Jpn. J. Med. Sci. Biol., 50, 113 - 121, 1997.

\title{
MOLECULAR TYPING OF VIBRIO VULNIFICUS ISOLATES BY RANDOM AMPLIFIED POLYMORPHIC DNA (RAPD) ANALYSIS
}

\author{
Dong Wook RYANG, Sang Won CHO, Myung Geun SHIN, Jong Hee SHIN, \\ and Soon Pal SUH
}

Department of Clinical Pathology, Chonnam University Medical School, HakDong 8, Dong-Gu, Kwangju 501-757, South Korea

(Received July 4, 1997. Accepted October 7, 1997)

SUMMARY: This study was undertaken to determine molecular types and genetic similarity among $V$. vulnificus isolates by RAPD analysis. We compared these results with serotypes of $V$. vulnificus. Ninety-seven $V$. vulnificus strains including 69 strains from Chonnam University Hospital (CUH; Kwangju, Korea), 13 from Wonkwang University Hospital (WUH; Iksan, Korea), 13 from the Japanese National Institute of Health (JNIH) and two reference strains (ATCC 33815 and ATCC 27562) were analyzed. Four molecular types comprising all the strains were obtained by RAPD analysis. Type I was the most common (60/95) and included 58 strains from CUH. Type I showed a further subdivision into seven subtypes. Type II (23/95) composed of 11 strains from CUH, nine from WUH, three from JNIH and two reference strains. Six type III strains comprised four WUH strains and two JNIH strains. All six strains of type IV were from JNIH. The range of genetic similarity values among $V$. vulnificus isolates was 0.24 to 1.00 . The serotypes of 95 strains were 04 (84.2\%), O14 (3.2\%), O1 (2.1\%), $013(2.1 \%)$, and $\mathrm{R}(2.1 \%)$. The most common $\mathrm{O} 4$ serotype strains were distributed among types I (60 strains), II (23 strains), III and IV (six strains). Although the $V$. vulnificus isolates showed a wide range of genetic similarity values, RAPD analysis could separate $V$. vulnificus strains into four molecular types, and the isolates from the same hospitals tended to belong to the same molecular type. There was no specific correlation between molecular types and serotypes of $V$. vulnificus. 


\section{INTRODUCTION}

DNA-based methods have recently been used for epidemiologic investigations of the bacterial strains. Among these molecular methods, restriction fragment length polymorphism (RFLP) has the advantage of being easy and rapid to perform, but it holds such disadvantages that the number of possible types is limited, with certain patterns appearing very commonly, so sometimes geographically distinct strains appear identical (1). Greater resolving power can be achieved by using a moderately repeated DNA sequence as a probe or by electrophoretic karyotyping by pulse-field gel electrophoresis (PFGE). But, the disadvantages of both of these procedures are the greater cost and time required (1). The random amplified polymorphic DNA (RAPD) assay that is based on the polymerase chain reaction (PCR) was introduced to overcome the above drawbacks of molecular methods for genetic studies (2). This method uses arbitrarily chosen 10-mer primers in conjunction with PCR, providing a variation of genomic DNA. Information is not required about flanking sequences of regions to be amplified in RAPD. Since each 10-mer primer screens 20 nucleotides for each amplified product, the polymorphisms are efficiently recognized (2).

Vibrio vulnificus infection is one of the most fatal diseases in Korea. This infection breaks out mainly in the southwestern coastal areas of Korea (3). There have been a lot of reports about the clinical manifestations and biologic characteristics of this infection, but molecular typing of $V$. vulnificus for epidemiological and genetic study hasn't been reported. Serotyping has been frequently used to differentiate $V$. vulnificus strains in Korea: most of $V$. vulnificus isolates have 04 serotype (4). But these $V$. vulnificus isolates disclosed various degrees of biochemical reaction profiles by the ATB 32GN system (API systems, La Balme-Les Grottes, France). These findings suggest that diverse biotypes exist in the same serotype (5).

For the epidemiological study of $V$. vulnificus infection in Korea, this study was based on $13 \mathrm{~V}$. vulnificus strains from Japanese National Institute of Health (JNIH), 82 Korean $V$. vulnificus isolates and two reference strains (ATCC 33815 and ATCC 27562). The purpose of this study was to determine molecular types and genetic similarity of $V$. vulnificus isolates by RAPD analysis and to compare these results with serotyping data. 


\section{MATERIALS AND METHODS}

V. vulnificus strains and serotyping: Ninety-seven $V$. vulnificus strains were analyzed in this study. They consisted of 69 strains (C1-C69) from Chonnam University Hospital (CUH; Kwangju, Korea), 13 (W14-W26) from Wongkwang University Hospital (WUH; Iksan, Korea), 13 (W1-W13) from JNIH and two reference strains ( $V$. vulnificus ATCC 27562 and ATCC 33815). Korean $V$. vulnificus strains were isolated from clinical specimens (blood and/or bulla) during past 12 years (1985-1996) at CUH and WUH. Blood and bulla specimens taken from patients were primarily cultured on blood agar and McConkey agar plates (BBL Microbiology System, Cockville, MD) at $36 \mathrm{C}$ for 18 to $24 \mathrm{hr}$. We used API 20E and ATB 32GN systems (API systems, La Balme-Les Grottes, France) to identify $V$. vulnificus from the isolated colonies. Serotyping was done by JNIH. The tube agglutination test was carried out with heated cell suspensions as used for preparation of antisera $(01-014, \mathrm{R})$. The results were read after incubation in a water bath at $50 \mathrm{C}$ for $18 \mathrm{hr}$.

RAPD analysis: $V$. vulnificus genomic DNA was extracted by the phenol/chloroform method (6). Twenty primers having 10-mer nucleotides (Operon 10-mer kit A; Operon Technologies, Atlanta, GA) were screened for their suitability in generating reproducible fingerprints that allowed discrimination among $V$. vulnificus isolates. Nine suitable primers were identified (Table I). The following protocols were used successfully to subtype strains of $V$. vulnificus.

$V$. vulnificus genomic DNA (20 ng) was added to $25 \mu \mathrm{l}$ of an amplification mixture containing $0.36 \mu \mathrm{M}$ operon primer, $1.5 \mathrm{mM} \mathrm{MgCl}_{2}, 10 \mathrm{mM}$ Tris- $\mathrm{HCl}(\mathrm{pH}$ 8.8), $10 \mathrm{mM} \mathrm{KCl}, 0.1 \%$ Triton X-100, $0.1 \mathrm{mM}$ dNTPs and 2 units of $T b r$ (Thermus brockianus) DNA polymerase (Dynazyme; Finzymes, Espoo, Finland). The reaction mixture was overlaid with two drops of sterile mineral oil to prevent evaporation. Thirty-five cycles of amplification were performed in a DNA thermal cycler (Model 480, Perkin-Elmer Cetus, CA). The time and temperature profiles of the reaction mixture were denaturation $5 \mathrm{sec}$ at $94 \mathrm{C}$, annealing $30 \mathrm{sec}$ at $36 \mathrm{C}$ and extension $60 \mathrm{sec}$ at $72 \mathrm{C}$. The products were visualized under ultraviolet light following electrophoreis of $10-\mu \mathrm{l}$ aliquots of the reaction product in ethidium bromide $(0.5 \mu \mathrm{l} / \mathrm{ml})$ stained with $1.5 \%$ agarose gel. We used $\Phi \mathrm{X} 174 \mathrm{RF}$ (Promega, Madison, WI) as a DNA size marker.

Statistical analysis of PCR results: We used the NTSYS-pc (numerical taxonomy system and multivariate analysis system, version 1.50, Applied Biostatistics Inc., CA) program to compose the phenogram for the differentiation of $V$. vulnificus strains. The genetic similarity was obtained from the distance values (D) by using the above program. The ' $D$ ' is expressed as $1-2$ Cxy / $(\mathrm{Ux}+\mathrm{Uy}+2 \mathrm{Cxy})$ in which Cxy equals the number of fragments in common to isolates $X$ and $Y$. Ux and $U y$ represent the number of unique bands in isolates $X$ and $\mathrm{Y}$, respectively. 
Table I. Sequence of primers used to amplify $V$. vulnificus DNA

\begin{tabular}{llll}
\hline Primer & $5^{\prime}$ to $3^{\prime}$ & Primer & $5^{\prime}$ to $3^{\prime}$ \\
\hline OPA-1 & CAGGCCCTTC & OPA-9 & GGGTAACGCC \\
OPA-2 & TGCCGAGCTG & OPA-12 & TCGGCGATAG \\
OPA-4 & AATCGGGCTG & OPA-17 & GACCGCTTGT \\
OPA-5 & AGGGGTCTTG & OPA-20 & GTTGCGATCC \\
OPA-7 & GAAACGGGTG & & \\
\hline
\end{tabular}

Table II. Molecular types of $V$. vulnificus isolates determined by RAPD analysis in comparison with serotype

\begin{tabular}{|c|c|c|c|c|c|c|c|c|c|c|c|}
\hline \multirow{3}{*}{ Serotype } & \multicolumn{10}{|c|}{ Molecular type } & \multirow{3}{*}{ Total $(\%)$} \\
\hline & \multicolumn{7}{|c|}{ I } & \multirow[t]{2}{*}{ II } & \multirow[t]{2}{*}{ III } & \multirow[t]{2}{*}{ IV } & \\
\hline & $\mathrm{a}$ & $\mathrm{b}$ & c & d & e & $\mathrm{f}$ & $\mathrm{g}$ & & & & \\
\hline 01 & & & & & & & & 2 & & & $2(2.1)$ \\
\hline 02 & 1 & & & & & & & & & & 1 \\
\hline 03 & & & & & & & & & & 1 & 1 \\
\hline 04 & & 25 & 11 & 18 & 2 & 1 & 1 & 17 & 3 & 2 & $80(84.2)$ \\
\hline 07 & & & & & & & & & & 1 & 1 \\
\hline 08 & & & & & & & & & & 1 & 1 \\
\hline 09 & & & & & & & & & & 1 & 1 \\
\hline 011 & & & & & & & & 2 & & & 1 \\
\hline 013 & & 1 & & & & & & 1 & & & $2(2.1)$ \\
\hline 014 & & & & & & & & 1 & 2 & & $3(3.2)$ \\
\hline $\mathrm{R}$ & & & & & & & & 1 & 1 & & $2(2.1)$ \\
\hline Total (\%) & & & & )$(63$ & & & & $3(24.2)$ & $6(6.3)$ & $6(6.3)$ & $95(100)$ \\
\hline
\end{tabular}

\section{RESULTS}

Serotyping V. vulnificus Isolates

Serotype 04 was the most prevalent: of 95 isolates, 80 strains (84.2\%) were serotype 04 . The serotypes of other 15 isolates were 014 (three strains, $3.2 \%$ ), 01 (two strains), $\mathrm{O} 13$ (two strains) and R (two strains). Serotypes O2, O3, O7, O8, 09 
and $\mathrm{O} 11$ represented only one strain each, and these strains came from JNIH (Table II).

\section{Molecular Typing by RAPD Patterns}

Each of $97 \mathrm{~V}$. vulnificus strains was analyzed with nine individual primers by the RAPD method. Nine primers used in this study were OPA-1, OPA-2, OPA4, OPA-5, OPA-7, OPA-9, OPA-12, OPA-17 and OPA-20. These were selected from 20 primers tested for their capacities to discriminate variability and their reproducibilities. All amplification was repeated at least twice. In each case, the most intense bands in the patterns were reproducible. Each primer generated between one and five major bands for individual strains. Examples of the patterns obtained with OPA-4 primer are presented in Fig. 1. The RAPD patterns by OPA-4 primer, having three to five bands between $300 \mathrm{bp}$ and 1,300 bp, represent the genetic diversity among the isolates (Fig. 1).

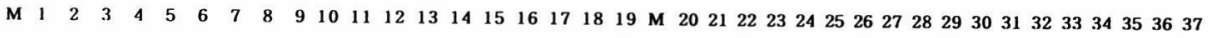

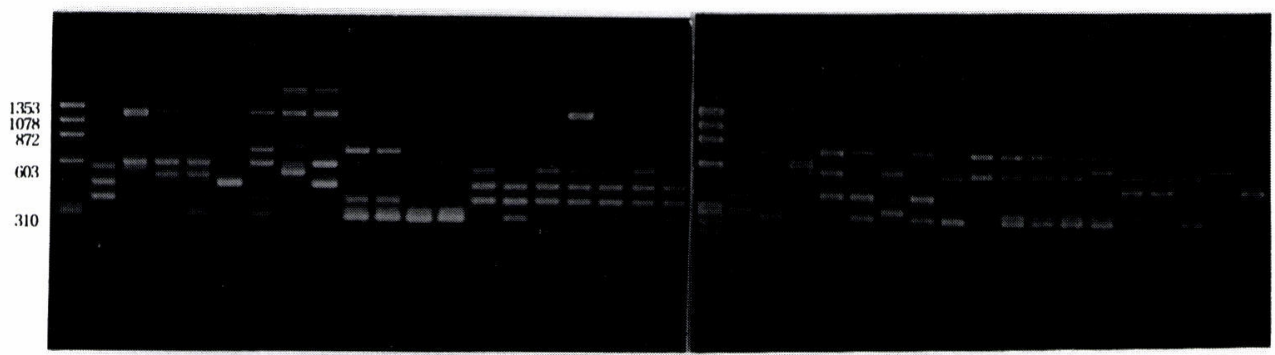

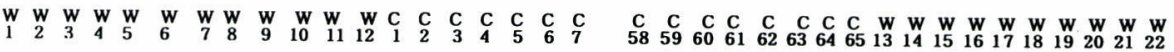

Fig. 1. RAPD profiles of $V$. vulnificus isolates with OPA-4 primer. Lane M, size maker (bp); lanes 1, 13-20, type I; lanes 9, 10, 21-37, type II; lanes 11, 12, type III; lanes 2-8, type IV.

All isolates were divided into four molecular types (I-IV) by RAPD analysis at a D value of $0.73 ; 60$ strains $(63.2 \%)$ belonged to type I, 23 strains (24.2\%) to type II, type III and IV included six strains. Type I strains were further separated into seven types (a-g). The isolates were grouped together as a single cluster at D 


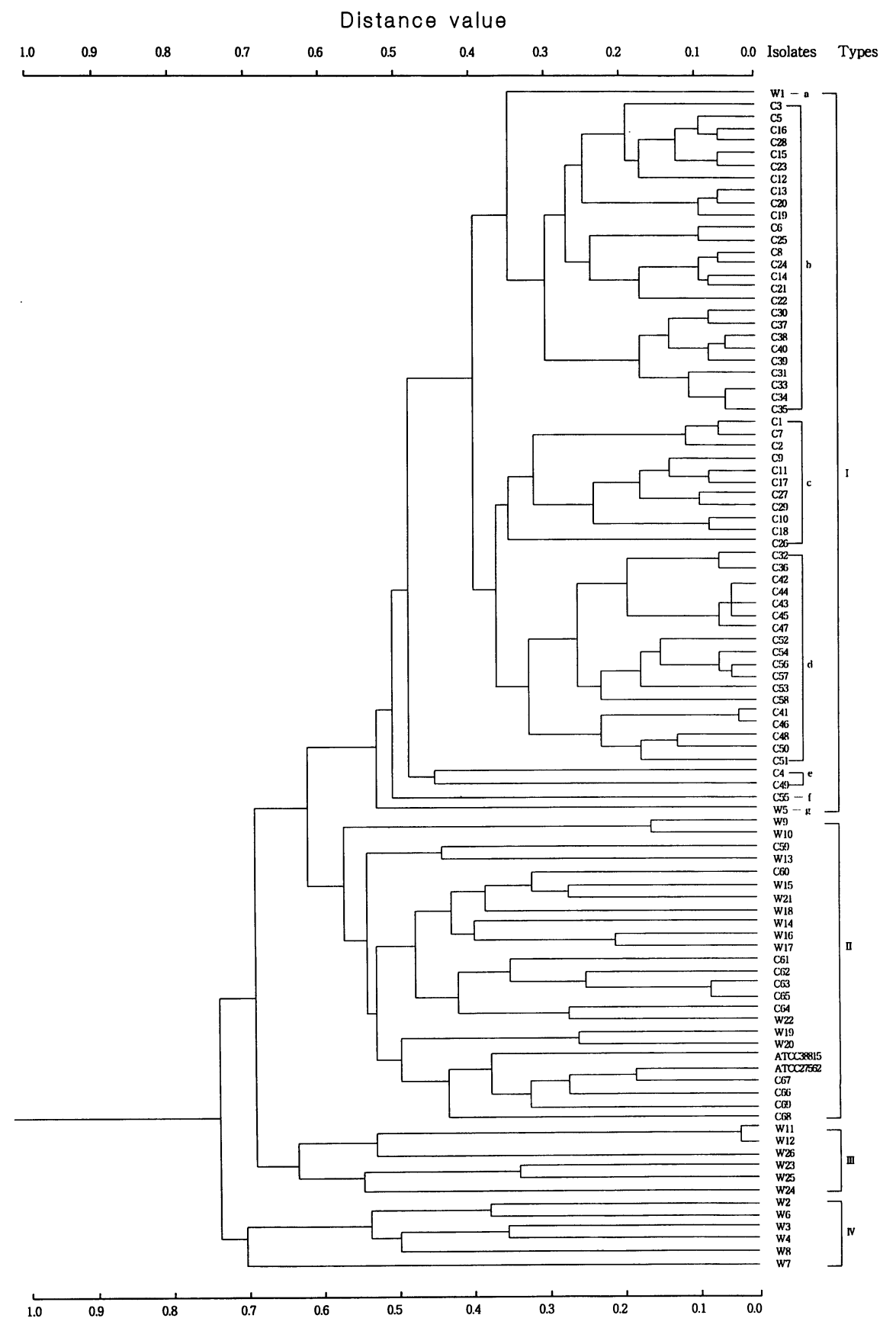

Fig. 2. Phenogram of 95 Vibrio vulnificus isolates by distance value 
Table III. Genetic similarity values of molecular types

\begin{tabular}{cc}
\hline Molecular type & Genetic similarity value \\
\cline { 2 - 2 } & Mean \pm SD \\
\hline I & $0.84 \pm 0.13$ \\
II & $0.66 \pm 0.14$ \\
III & $0.55 \pm 0.25$ \\
IV & $0.48 \pm 0.17$ \\
\hline
\end{tabular}

of 0.73. Type I strains, except for two strains (W1 and W2), mainly came from CUH (58 strains); $84.1 \%$ (58/69) of CUH strains were distributed into type I. Fifty-eight of type I strains from CUH were isolated during 1985-1996. Type II included reference strains of $V$. vulnificus ATCC 33815 and 27562. The strains of type II originated from CUH (11 strains), WUH (nine strains) and JNIH (three strains). Type II 11 strains from CUH were isolated in 1987 (two strains), 1990 (one strain), 1991 (three strains), 1994 (three strains) and 1995 (two strains). Four WUH and two JNIH strains composed of type III. The six strains of type IV were from JNIH (Fig. 2).

\section{Genetic Similarity}

The distribution of genetic similarity values was 0.24 to 1.00 in all isolates, thus the isolates had a wide range of similarity values. The mean similarity values of all isolates, types I, II, III and IV, were $0.76,0.84,0.66,0.55$ and 0.48 , respectively (Table III).

\section{Relation between Serotypes and RAPD Types}

The most common 04 serotype strains were distributed into RAPD type I (58 strains), II (17 strains), III (three strains) and IV (two strains). The strains having 014 serotype were divided into type III (two strains) and II (one strain). Serotype 013 strains belonged to types I and II; R serotype to type II and III. Type IV included 03, 07, 08 and 09 serotype strains. The relationship between serotype and RAPD type was represented in Table II. 


\section{DISCUSSION}

$V$. vulnificus is a virulent Vibrio species associated with wound infection after exposure to contaminated sea water and primary septicemia following consumption of contaminated sea food. Serotypes of $V$. vulnificus can be differntiated based on $O$ antigen. The 15 serotypes (O1-O14, and R) are defined by Shimada and Sakazaki (7). The common serotypes of $V$. vulnificus isolated from human specimens are known to be $\mathrm{O} 4$ and 01 . The $\mathrm{O} 4$ serotype was the most common among $V$. vulnificus isolates from Korea.

RAPD analysis of $V$. vulnificus strains showed a high-level genetic diversity in this study. The range of genetic similarity values among $V$. vulnificus isolates was 0.24 to 1.00 . The diversity of $V$. vulnificus isolates was noted in the previous studies of cellular fatty acid (CFA) composition and restriction fragment length polymorphism (RFLP) $(8,9)$. In the study of RFLP of the $V$. vulnificus strains (8), the same serotype showed diverse patterns. Numerical analysis of CFA data can separate 95 clinical $V$. vulnificus strains isolated from Korea into nine subgroups (I-IX): the $\mathrm{O} 4$ serotype strains are also distributed into subgroups VII (40 strains), V (27 strains), III (four strains), I (two strains), IV (two strains) and VI (one strain) (9). These findings suggest that various genotypes or biotypes may be present in the same serotype.

The four molecular RAPD types were separated at a $\mathrm{D}$ value of about 0.73 . The RAPD type I strains, the most common type, consisted of CUH strains except two strains isolated from JNIH. WUH strains were distributed only in type II (nine strains) and III (four strains). Therefore, these data reflected relationships between RAPD types and isolated areas, especially in CUH and WUH strains. But there was no definitive relationship between isolated date and molecular types because type I and type II strains from CUH were isolated during 19851996.

The serotypes of 95 strains were $04(84.2 \%), 014(3.2 \%), 01(2.1 \%), 013$ $(2.1 \%)$ and $\mathrm{R}(2.1 \%)$ in order of frequency. The $\mathrm{O} 4$ serotype represented $>90 \%$ of all Korean strains in this study. The Korean strains of 04 serotype (82 strains) were distributed among RAPD type I (58 strains), type II (20 strains) and type III (four strains). These RAPD patterns were not related to the serotypes, while RAPD was more discriminative than serotyping.

In summary, V. vulnificus strains disclosed a high level of genetic diversity, separated into four molecular types by RAPD analysis. Also, the isolates from different hospitals had a tendency to belong to different molecular types. These 
findings support the conclusion that RAPD typing of $V$. vulnificus strains may provide useful epidemiological information and overcome the limitation of serotyping of $V$. vulnificus in Korea.

\section{REFERENCES}

1. Bostock, A., Khattak, M. N., Matthews, R. and Burnie, J. (1993): Comparison of PCR fingerprinting, by random amplification of polymorphic DNA, with other molecular typing methods of Candida albicans. J. Gen. Microbiol., 139, 2179-2184.

2. Williams, J. G. K., Kubelik, A. R., Livak, K. J., Rafalski, J. A. and Tingey, S. V. (1990): DNA polymorphisms amplified by arbitrary primers are useful as genetic markers. Nuc. Acids Res., 18, 6531-6535.

3. Shin, M. G., Shin, J. H. and Ryang, D. W. (1993): Clinical characteristics of Vibrio vulnificus infection. Korean J. Clin. Pathol., 13, 287-293.

4. Kim, S. M., Park, Y. and Chong, Y. S. (1991): A study on biological characteristics and serovar of Vibrio vulnificus isolated in Korea. J. Korean Soc. Microbiol., 26, 403-415.

5. Shin, J. H., Shin, M. G. and Ryang, D. W. (1993): Evaluation of ATB 32GN system for identification of Vibrio vulnificus. Korean J. Clin. Pathol., 13, 281-286.

6. Wang, G., Whittam, T. S., Berg, C. M. and Berg, D. E. (1993): RAPD (arbitrary primer) PCR is more sensitive than multilocous enzyme electrophoresis for distinguishing related bacterial strains. Nuc. Acids Res., 21, 5930-5933.

7. Shimada, T. and Sakazaki, R. (1984): On the serology of Vibrio vulnificus. Jpn. J. Med. Sci. Biol., 37, 241-246.

8. Cho, J. H., Ryang, D. W. and Moon, Y. H. (1995): Restriction enzyme analysis of chromosomal DNA and serotype of Vibrio vulnificus prevailed in Honam area. Korean J. Clin. Pathol., 15, 240-249.

9. Shin, M. G., Shin, J. H., Suh, S. P., Ryang, D. W. and Bae, K. S. (1997): Subgrouping of Vibrio vulnificus by celluar fatty acid analysis. J. Korean Soc. Microbiol., 32, 27-37. 